\title{
Growth index of matter perturbations in the light of Dark Energy Survey
}

\author{
Spyros Basilakos ${ }^{1,2, a}$, Fotios K. Anagnostopoulos ${ }^{3, b}$ \\ ${ }^{1}$ Research Center for Astronomy and Applied Mathematics, Academy of Athens, Soranou Efesiou 4, 11527 Athens, Greece \\ ${ }^{2}$ National Observatory of Athens, V. Paulou and I. Metaxa, 15236 Penteli, Greece \\ ${ }^{3}$ Department of Physics, National and Kapodistrian University of Athens, Zografou Campus GR 157 73, Athens, Greece
}

Received: 9 December 2019 / Accepted: 22 February 2020 / Published online: 6 March 2020

(C) The Author(s) 2020

\begin{abstract}
We study how the cosmological constraints from growth data are improved by including the measurements of bias from Dark Energy Survey (DES). In particular, we utilize the biasing properties of the DES Luminous Red Galaxies (LRGs) and the growth data provided by the various galaxy surveys in order to constrain the growth index $(\gamma)$ of the linear matter perturbations. Considering a constant growth index we can put tight constraints, up to $\sim 10 \%$ accuracy, on $\gamma$. Specifically, using the priors of the Dark Energy Survey and implementing a joint likelihood procedure between theoretical expectations and data we find that the best fit value is in between $\gamma=0.64 \pm 0.075$ and $0.65 \pm 0.063$. On the other hand utilizing the Planck priors we obtain $\gamma=0.680 \pm 0.089$ and $0.690 \pm 0.071$. This shows a small but non-zero deviation from General Relativity $\left(\gamma_{\mathrm{GR}} \approx 6 / 11\right)$, nevertheless the confidence level is in the range $\sim 1.3-2 \sigma$. Moreover, we find that the estimated mass of the dark-matter halo in which LRGs survive lies in the interval $\sim 6.2 \times 10^{12} h^{-1} M_{\odot}$ and $1.2 \times 10^{13} h^{-1} M_{\odot}$, for the different bias models. Finally, allowing $\gamma$ to evolve with redshift [Taylor expansion: $\gamma(z)=\gamma_{0}+\gamma_{1} z /(1+z)$ ] we find that the $\left(\gamma_{0}, \gamma_{1}\right)$ parameter solution space accommodates the GR prediction at $\sim 1.7-2.9 \sigma$ levels.
\end{abstract}

\section{Introduction}

The past and present analysis of various cosmological data (SNIa, Cosmic Microwave Background-CMB, Baryonic Acoustic Oscillations-BAOs, Hubble parameter measurements etc) converge to the following cosmological paradigm, the observed Universe is spatially flat and the cosmic fluid consists of $\sim 4 \%$ luminous (baryonic) matter, $\sim 26 \%$ dark

\footnotetext{
a e-mail: svasil@academyofathens.gr

b e-mail: fotis-anagnostopoulos@ hotmail.com (corresponding author)
}

matter and $\sim 70 \%$ some sort of dark energy (hereafter DE) which plays a key role in explaining the accelerated expansion of the universe (cf. [2, 13, 15, 24,31] and references therein). Despite the fact that there is an agreement among the majority of cosmologists concerning the ingredients of the cosmic fluid however, there are different explanations regarding the physical mechanism which causes the accelerated expansion of the universe. In brief, the general avenue that one can design in order to study cosmic acceleration is to treat DE either as a new field in nature or as a modification of General Relativity (see for a review [4, 18,20]).

It has been proposed $[7,38,57]$ that in order to discriminate between scalar field DE and modified gravity one may utilize the evolution of the linear growth of matter fluctuations $\delta_{m}(z)=\delta \rho_{m} / \rho_{m}$. In particular, we introduce the growth rate of clustering, which is given by $f(a)=\frac{d \ln D}{d \ln a} \simeq \Omega_{m}^{\gamma}(a)$, where $D(a)=\delta_{m}(a) / \delta_{m}(a=1)$ is the linear growth factor (normalized to unity at the present epoch), $a(z)=(1+z)^{-1}$ is the scale factor of the universe, $\Omega_{m}(a)$ is the dimensionless matter density parameter and $\gamma$ is the so called growth index [45,65].

In fact the determination of the growth index is considered one of the main targets in these kind of studies because it can be used in order to test general relativity (GR) on extragalactic scales [28], even in a model independent fashion [43]. Indeed, in the literature one may find a large family of studies in which the functional form of the growth index is given analytically for several cosmological models namely, scalar field [11,26,30,39,42,52,66], $f(R)[27,61], f(T)[6]$ Finsler-Randers [12], running vacuum models [10], clustered and Holographic dark energy [41].

From the view point of large scale structure, the study of the distribution of matter on extragalactic using different mass tracers (galaxies, AGNs, clusters of galaxies etc) provides important constraints on theories of structure formation. Specifically, owing to the fact that gravity reflects, 
via gravitational instability, on the physics of clustering [45] it is natural to utilize the clustering/biasing properties of the extragalactic mass tracers in constraining cosmological models (see $[8,36,40])$ as well as to test the validity of GR on cosmological scales (see [11,32]). Following the above lines, in the current article we combine the linear bias data of Luminous Red Galaxies (hereafter LRGs; [23]), recently released by the DES group, with the growth rate data as provided by Sagredo et al. [48], in order to place constraints on $\left(\gamma, M_{h}\right)$. Notice that $M_{h}$ is the dark matter halo in which LRG live.

The structure of the paper is as follows. In Sect. 2 we present the DES bias data and the growth data. In Sect. 3 we provide the family of basic bias models, while in Sect. 4 we discuss the evolution of linear matter fluctuations. The outcome of our analysis is presented in Sect. 5, while our main conclusions can be found in Sect. 6 .

\section{DESY1 red galaxies bias data and growth data}

In a sequence of previous theoretical articles we have proposed to use the biasing properties of extragalactic sources in order to constrain the growth index of matter fluctuations [11]. Therefore, in the light of recent Dark Energy Survey (DES) bias data, we attempt to compare the predictions of the most popular linear bias models (see below) with the data. Specifically, the DES bias data [23] were extracted in the context of the angular correlation function (ACF) using the 1-year DES sample of $\sim 6.6 \times 10^{5}$ LRGs as tracers of the LSS. In Table 1 we list the numerical values of the DES bias data with the corresponding errors.

The aim of our work is the following: if we accept that the background expansion is given by the concordance $\Lambda \mathrm{CDM}$ model then we are interested to check the growth index of matter fluctuations. Specifically, we restrict the present analysis to the most popular expansion models. First we utilize the DES/Planck/JLA/BAO $\Lambda$ CDM cosmology, namely $\Omega_{m 0}=1-\Omega_{\Lambda 0}=0.301, h=0.682, \Omega_{b 0}=0.048$, $n=0.973$ with $\sigma_{8}=0.801$ [1] and second we use the Planck TT+TE+EE+low+lensing $\Lambda$ CDM cosmology, hence $\Omega_{m 0}=1-\Omega_{\Lambda 0}=0.3153, h=0.6736, \Omega_{b 0}=0.0493$, $n=0.9649$, and $\sigma_{8}=0.811$ [2]. In this context, the normalized Hubble parameter of the $\triangle \mathrm{CDM}$ model is written as

$E(z)=\left[\Omega_{m 0}(1+z)^{3}+\Omega_{\Lambda 0}\right]^{1 / 2}$.

In addition to DES bias data, we use in our analysis the growth data and the corresponding covariances as collected by Sagredo et al. [48] (see their Table 1 and references therein). This sample contains 22 entries for which the product $f(z) \sigma_{8}(z)$ is available as a function of redshift, where
Table 1 The measured bias data of the 1-year DES LRGs from [23]

\begin{tabular}{lll}
\hline Red. Range & Median Redshift & DESY1 bias \\
\hline $0.15<z<0.3$ & $0.225 \pm 0.075$ & $1.40 \pm 0.077$ \\
$0.3<z<0.45$ & $0.375 \pm 0.075$ & $1.61 \pm 0.051$ \\
$0.45<z<0.6$ & $0.525 \pm 0.075$ & $1.60 \pm 0.040$ \\
$0.6<z<0.75$ & $0.675 \pm 0.075$ & $1.93 \pm 0.045$ \\
$0.75<z<0.9$ & $0.825 \pm 0.075$ & $1.99 \pm 0.066$ \\
\hline
\end{tabular}

$f(z)$ is the growth rate of clustering. ${ }^{1}$ It is well known that the product $f \sigma_{8}$ is almost a model-independent parametrization of expressing the observed growth history of the universe [55].

\section{Bias models}

Let us first briefly present the main bias models. In particular, from the so called merging bias family we include here the models of Sheth et al. [51], Jing [34] and De Simone et al. [21].

For these models the bias factor is written as a function of the peak-height parameter, $v=\delta_{c}(z) / \sigma\left(M_{h}, z\right)$ where $\delta_{c}$ is the linearly extrapolated density threshold above which structures collapse. Here we use the accurate fitting formula of Weinberg and Kamionkowski [67] to estimate $\delta_{c}(z)$. Moreover, the mass variance is written as

$\sigma\left(M_{h}, z\right)=\left[\frac{D^{2}(z)}{2 \pi^{2}} \int_{0}^{\infty} k^{2} P(k) W^{2}(k R) d k\right]^{1 / 2}$

where $W(k R)=3[\sin (k R)-k R \cos (k R)] /(k R)^{3}$ is the top-hat smoothing kernel with $R=\left[3 M_{h} /\left(4 \pi \rho_{m}\right)\right]^{1 / 3}, M_{h}$ is the halo mass and $\rho_{m}$ is the present value of the mean matter density, namely $\rho_{m} \simeq 2.78 \times 10^{11} \Omega_{m} M_{\odot} / \mathrm{Mpc}^{3}$. The quantity $P(k)$ is the CDM linear power spectrum given by $P(k)=P_{0} k^{n} T^{2}(k)$ where $n$ is the spectral index of the primordial power spectrum and $T(k)$ is the CDM transfer function provided by Eisenstein and $\mathrm{Hu}$ [22]:

$T(k)=\frac{L_{0}}{L_{0}+C_{0} q^{2}}$

\footnotetext{
${ }^{1}$ By definition the estimator $f(z) \sigma_{8}(z)$ is independent from linear bias and thus it is not affected by the dark matter halo. Indeed, the observed growth rate of structure $\left(f_{\mathrm{obs}}(z)=\beta b\right)$ is derived from the redshift space distortion parameter $\beta(z)$ and the linear bias. Observationally, using the anisotropy of the spatial correlation function one can estimate the $\beta(z)$ parameter. The linear bias factor can be defined as the ratio of the variances of the tracer (galaxies, Luminous Red Galaxies etc) and underlying mass density fields, smoothed at $8 h^{-1} \mathrm{Mpc} b(z)=\sigma_{8, \operatorname{tr}}(z) / \sigma_{8}(z)$, where $\sigma_{8, \text { tr }}(z)$ is measured directly from the sample. Combining the above definitions we arrive at $f \sigma_{8}=\beta \sigma_{8}$,tr, hence the growth rate data are not not affected from bias, implying that the two data sets used in the present analysis are not correlated.
} 
with $L_{0}=\ln (2 e+1.8 q), e=2.718, C_{0}=14.2+\frac{731}{1+62.5 q}$ and $q=k / \Gamma$ with $\Gamma$ being is the shape parameter given by Sugiyama [58]:

$\Gamma=\Omega_{m} h \exp \left(-\Omega_{b}-\sqrt{2 h} \frac{\Omega_{b}}{\Omega_{m}}\right)$.

Taking the aforementioned quantities into account and using Eq. (2) the normalization of the power spectrum becomes

$P_{0}=2 \pi^{2} \sigma_{8}^{2}\left[\int_{0}^{\infty} T^{2}(k) k^{n+2} W^{2}\left(k R_{8}\right) d k\right]^{-1}$

where $\sigma_{8} \equiv \sigma\left(R_{8}, 0\right)$.

Below we provide some details concerning the bias models.

\subsection{SMT and JING}

Sheth et al. ([51], hereafter SMT) based on the ellipsoidal collapse model they found the following bias formula $b(v)=1+\frac{1}{\sqrt{\alpha}} \delta_{c}(z)\left[\sqrt{\alpha}\left(\alpha v^{2}\right)+\sqrt{\alpha} b\left(\alpha v^{2}\right)^{1-c}-f(v)\right]$

with

$f(v)=\frac{\left(\alpha v^{2}\right)^{c}}{\left(\alpha v^{2}\right)^{c}+b(1-c)(1-c / 2)}$.

Using N-body simulations they evaluated the free parameters of the model, $\alpha=0.707, b=0.5, c=0.6$

Also, Jing [34] proposed the following bias form

$b(v)=\left(\frac{0.5}{v^{4}}+1\right)^{0.06-0.02 v}\left(1+\frac{v^{2}-1}{\delta_{c}}\right)$.

\subsection{DMR}

De Simone et al. [21] (hereafter DMR) generalized the original Press-Schether formalism incorporating a nonMarkovian extension with a stochastic barrier. In this model, the critical value for spherical collapse was assumed to be a stochastic variable, whose scatter reflects a number of complicated aspects of the underlying dynamics. Therefore, the bias factor is

$$
\begin{aligned}
b(v)= & 1+\sqrt{\alpha} \frac{v^{2}}{\delta_{c}}\left[1+0.4\left(\frac{1}{\alpha v^{2}}\right)^{0.6}\right] \\
- & \frac{1}{\sqrt{\alpha} \delta_{c}\left[1+0.067\left(\frac{1}{\alpha \nu^{2}}\right)^{0.6}\right]}
\end{aligned}
$$

\subsection{BPR}

In addition to merging bias models we shall use the generalized model of Basilakos et al. [11] (hereafter BPR). This form of bias is valid for any dark energy model including those of modified gravity. In this case, using the hydrodynamic equations of motion, linear perturbation theory and the Friedmann-Lemaitre solutions a second differential equation of bias is derived Basilakos et al. [11]. The solution of the differential equation is given by:

$b(z)=1+\frac{b_{0}-1}{D(z)}+C_{2} \frac{J(z)}{D(z)}$

with $J(z)=\int_{0}^{z} \frac{1+y}{E(y)} d y$ where $b_{0}$ is the bias factor at the present time. The integration constants $b_{0}$ and $C_{2}$ can be found in Basilakos et al. [11], namely

$b_{0}=0.857\left[1+\left(\frac{\Omega_{m}}{0.27} \frac{M_{h}}{10^{14} h^{-1} M_{\odot}}\right)^{0.55}\right]$

and

$C_{2}=1.105\left(\frac{\Omega_{m}}{0.27} \frac{M_{h}}{10^{14} h^{-1} M_{\odot}}\right)^{0.255}$.

Finally, we would like to mention that the performance of the above bias models in reproducing the DES bias data has been studied in Papageorgiou et al. [44]. At this point some comments are in order. The choice of linear bias, used in the current work, is dictated by the fact that the DES bias data were extracted in the linear bias regime. According to Krause et al. [35] the scale of $\sim 8 h^{-1} \mathrm{Mpc}$ utilized by the DES team implies that the impact of non-linear effects on clustering and thus on biasing is almost negligible (see also [23]). In this context, we use the common assumption that each extragalactic mass tracers is hosted by a dark matter halo of a given mass. Possible double tracer occupancy of the dark matter halo is ignored. It is interesting to mention here that the linear bias approach (also used by DES team) relates a mass tracer, being a galaxy, an AGN, a LRG or a cluster of galaxies, with a host dark matter halo within which the mass tracer forms and evolves. The bias models themselves follow the linear evolution of the host halo and not the internal evolution of the astrophysical processes of the tracer. Thus the assumption is that the effects of nonlinear gravity and hydrodynamics (merging, feedback mechanisms, etc.) can be ignored in the linear-regime (see [19,25]).

However, in our work following the notations of [44] we include in our bias analysis the nonlinear power spectrum of [60] (and references therein). Notice that the halo-fit model of [60] is applicable in the wave-number range of $k<30 \mathrm{hpcc}^{-1}$ and the redshift range of $0 \leq z \leq 10$. In brief, the nonlinear power spectrum consists of two terms: $\Delta^{2}(k, z)=\Delta_{H}^{2}(k, z)+\Delta_{Q}^{2}(k, z)$, where $\Delta^{2}(k)$ is the dimensionless nonlinear power spectrum $\Delta^{2}(k)=k^{3} P(k) /\left(2 \pi^{2}\right)$. The first term in the above formula is the so called one-halo term which dominates the power spectrum at small scales and it describes the matter correlation within the same dark matter halo. Also, the second is the two-halo term, which dominates 
the power spectrum at large scales and it describes the matter correlation between two distinct halos (for more details see appendix).

\section{Evolution of liner growth}

In this section we discuss the main points of the linear growth of matter fluctuations via which the growth index, $\gamma$, enters in the current analysis. Focusing on sub-horizon scales the differential equation that governs the linear matter perturbations $[39,56,61,62]$ and references therein) is given by

$\ddot{\delta}_{m}+2 H \dot{\delta}_{m}=4 \pi G_{\text {eff }} \rho_{m} \delta_{m}$,

where $\rho_{m} \propto a^{-3}$ is the matter density, $G_{\text {eff }}=G_{N} Q(t)$ with $G_{N}$ being the Newton's gravitational constant, while the effects of modified gravity are encapsulated in the quantity $Q(t)$. Of course for those DE models which adhere to General Relativity $G_{\text {eff }}$ reduces to $G_{N}$, hence $Q(a)=1$.

The solution of the aforementioned equation (13) is $\delta_{m} \propto$ $D(a)$, where $D(a)$ is the growth factor. For any type of gravity the growth rate of clustering is given by the following useful parametrization $[39,45,65]$

$f(a)=\frac{d \ln \delta_{m}}{d \ln a} \simeq \Omega_{m}^{\gamma}(a)$

and thus we have

$D(a)=\exp \left[\int_{1}^{a(z)} \frac{\Omega_{m}^{\gamma}(y)}{y} d y\right]$,

where $\Omega_{m}(a)=\Omega_{m 0} a^{-3} / E^{2}(a)$ and $\gamma$ is the growth index. Notice that the growth factor is normalized to unity at the present epoch.

Now, inserting the operator $d / d t=H d / d \ln a$ and Eq. (14) into Eq. (13) we arrive at

$\frac{d f}{d \ln a}+f^{2}+\left(\frac{\dot{H}}{H^{2}}+2\right) f=\frac{3}{2} Q(a) \Omega_{m}(a)$.

Considering the concordance $\Lambda$ CDM model, namely $Q(a)=$ 1 it is easy to show that

$\frac{\dot{H}}{H^{2}}+2=\frac{1}{2}-\frac{3}{2} \mathrm{w}(a)\left[1-\Omega_{m}(a)\right]$,

where $w(a)=-1$. In this case the Hubble parameter $H(a)=H_{0} E(a)$, where $E(a)$ is given by Eq. (1) and $H_{0}$ is the Hubble constant. ${ }^{2}$

Generally speaking the growth index may not be a constant but rather evolve with redshift; $\gamma \equiv \gamma(z)$. In this framework,

\footnotetext{
${ }^{2}$ For the comoving distance and for the dark matter halo mass we use the traditional parametrization $H_{0}=100 \mathrm{hkm} / \mathrm{s} / \mathrm{Mpc}$. Of course, when we treat the power spectrum shape parameter $\Gamma$ we utilize $h \equiv \tilde{h}=0.68$ [1].
}

substituting Eq. (14) into Eq. (16) we find

$$
\begin{aligned}
& -(1+z) \gamma^{\prime} \ln \left(\Omega_{m}\right)+\Omega_{m}^{\gamma}+3 \mathrm{w}\left(1-\Omega_{m}\right)\left(\gamma-\frac{1}{2}\right) \\
& +\frac{1}{2}=\frac{3}{2} Q \Omega_{m}^{1-\gamma}
\end{aligned}
$$

where the prime denotes derivative with respect to redshift. In the present work we restrict our analysis to the following two cases $[5,9,16,46]$ :

$\gamma(z)= \begin{cases}\gamma_{0}, & \Gamma_{1} \text {-parametrization } \\ \gamma_{0}+\gamma_{1} z /(1+z), & \Gamma_{2} \text {-parametrization }\end{cases}$

Using the latter $\Gamma_{2}$-parametrization, which is nothing else but a Taylor expansion around $a(z)=1$, together with Eq. (18) evaluated at the present time $(z=0)$, we can write the parameter $\gamma_{1}$ in terms of $\gamma_{0}$

$\gamma_{1}=\frac{\Omega_{m 0}^{\gamma_{0}}+3 \mathrm{w}_{0}\left(\gamma_{0}-\frac{1}{2}\right)\left(1-\Omega_{m 0}\right)-\frac{3}{2} Q_{0} \Omega_{m 0}^{1-\gamma_{0}}+\frac{1}{2}}{\ln \Omega_{m 0}}$.

At large redshifts $(z \gg 1) \Omega_{\Lambda}(z) \simeq 0$ the asymptotic value of the growth index becomes $\gamma_{\infty}=\gamma_{0}+\gamma_{1}$. In general, plugging $\gamma_{0}=\gamma_{\infty}-\gamma_{1}$ into Eq. (20) we can define the constants $\gamma_{1}$ as a function of $\left(\Omega_{m 0}, \gamma_{\infty}, w_{0}, Q_{0}\right)$. For examble, in the case of $\Omega_{m 0}=0.301, \gamma_{\infty} \simeq 6 / 11, w_{0}=-1$ and $Q_{0}=1$, the above calculations give $\gamma_{0}^{(t h)} \simeq 0.556 . \gamma_{1}^{(t h)} \simeq-0.011$.

\section{The likelihood analysis}

In this section we provide the statistical method that we adopt in order to constrain the growth index, presented in the previous section. We implement a standard $\chi^{2}$ minimization analysis in order to constrains either the $\left(\gamma, M_{h}\right)$ parameter space. Specifically, in our case the situation is as follows:

(1) For the DES biasing cosmological probe we use

$\chi_{\mathrm{DES}}^{2}\left(\mathbf{p}_{1}\right)=\sum_{i=1}^{5}\left[\frac{b_{\mathrm{obs}}\left(z_{i}\right)-b_{t h}\left(z_{i}, \mathbf{p}_{1}\right)}{\sigma_{b i}}\right]^{2}$

where the various forms of the theoretical $b_{\text {th }}$ are given in Sect. 3. Notice that $\sigma_{b i}=\sqrt{\sigma_{i}^{2}+\sigma_{z}^{2}}$, where $\sigma_{i}$ and $\sigma_{z}=$ 0.075 are the uncertainties of the observed bias and redshift respectively (see Table 1).

2. Regarding the analysis of the growth-rate data we use

$\chi_{\mathrm{gr}}^{2}\left(\mathbf{p}_{2}\right)=\mathbf{M C}_{\mathrm{cov}}^{-1} \mathbf{M}^{T}$

where $\mathbf{M}=\left\{f \sigma_{8, \mathrm{obs}}\left(z_{1}\right)-f \sigma_{8}\left(z_{1}, \mathbf{p}_{2}\right), \ldots, f \sigma_{8, \mathrm{obs}}\left(z_{n}\right)-\right.$ $\left.f \sigma_{8}\left(z_{n}, \mathbf{p}_{2}\right)\right\}$ and $\mathbf{C}_{\mathrm{cov}}^{-1}$ is the inverse covariance matrix [48]. The theoretical growth-rate is given by:

$f \sigma_{8}\left(z, \mathbf{p}_{2}\right)=\sigma_{8} D(z) \Omega_{m}(z)^{\gamma(z)}$. 
The vectors $\mathbf{p}_{1}$ and $\mathbf{p}_{2}$ provide the free parameters that enter in deriving the theoretical expectations. The first vector includes the free parameters which are related to the expansion and the environment of the parent dark matter halo in which the LRGs DES galaxies live. Specifically, for constant $\gamma$ we have $\mathbf{p}_{1}=\left\{\mathbf{p}_{2}, M_{h}\right\}=\left\{\Omega_{m 0}, h, \sigma_{8}, \gamma, M_{h}\right\}$, while for the case of evolving $\gamma$, the vector is defined as: $\mathbf{p}_{1}=$ $\left\{\Omega_{m 0}, h, \sigma_{8}, \gamma_{0}, \gamma_{1}, M_{h}\right\}$. We remind the reader that the cosmological parameters $\left\{\Omega_{m 0}, h, \sigma_{8}\right\}=\{0.301,0.682,0.801\}$ are given in Sect. 2 [1].

Since we wish to perform a joint likelihood analysis of the two cosmological probes and owing to the fact that likelihoods are defined as $\mathcal{L}_{i} \propto \exp \left(-\chi_{i}^{2} / 2\right)$, the overall likelihood function becomes

$\mathcal{L}\left(\mathbf{p}_{1}\right)=\mathcal{L}_{\text {DES }} \times \mathcal{L}_{\text {gr }}$,

which is equivalent to:

$\chi^{2}\left(\mathbf{p}_{1}\right)=\chi_{\mathrm{DES}}^{2}+\chi_{\mathrm{gr}}^{2}$.

Based on the above we will provide our results for each free parameter that enters in the $\mathbf{p}_{1}$ vector. The uncertainty of each fitted parameter will be estimated after marginalizing one parameter over the other, providing as its uncertainty the range for which $\Delta \chi^{2}(\leq 1 \sigma)$.

As a further quality measure over the fits, we have used the AIC [3] criterion, in a modified form that is appropriate for small data sets [37]. Considering Gaussian errors AIC is given by

$\mathrm{AIC}=-2 \ln \mathcal{L}_{\max }+2 k+\frac{2 k(k+1)}{N-k-1}$,

where $N$ is the total number of data and $k$ is the number of fitted parameters (see also [37]). Of course, a smaller value of AIC implies a better model-data fit. In order to test the performance of the different bias models in fitting the data we need to utilize the model pair difference, namely $\triangle \mathrm{AIC}=$ $\mathrm{AIC}_{\text {model }}-\mathrm{AIC}_{\min }$. From one hand, the restriction $\triangle \mathrm{AIC} \leq$ 2 indicates consistency between the two comparison models. On the other hand, the inequalities $4<\Delta$ AIC $<7$ indicate a positive evidence against the model with higher value of $\mathrm{AIC}_{\text {model }}$ Burnham and Anderson [17], while the condition $\Delta \mathrm{AIC} \geq 10$ suggests a strong such evidence.

\subsection{Observational constraints}

Below, we provide a qualitative discussion of our constraints, giving the reader the opportunity to appreciate the new results of the current study.

\subsubsection{Constant growth index}

Here we focus on the $\Gamma_{1}$ parametrization, which means that the parameter space contains the following free parameters $\left(\gamma, M_{h}\right)$. The presentation of our constraints is provided in Table 2 for the case of DES/Planck/JLA/BAO reference cosmology (see Sect. 2). The Table includes the goodness of fit statistics $\left(\chi_{\min }^{2}, A I C\right)$, for the specific bias models. Also, in Fig. 1 we present the $1 \sigma, 2 \sigma$ and $3 \sigma$ confidence contours in the $\left(\gamma, M_{h}\right)$ plane.

In particular, we find:

- For SMT model: $\chi_{\min }^{2}=15.042(\mathrm{AIC}=19.542), \gamma=$ $0.640 \pm 0.071$ and $\log \left(M_{h} / h^{-1} M_{\odot}\right)=13.000 \pm 0.072$.

- For JING model: $\chi_{\min }^{2}=15.975(\mathrm{AIC}=20.475), \gamma=$ $0.650 \pm 0.063$ and $\log \left(M_{h} / h^{-1} M_{\odot}\right)=12.910 \pm 0.062$.

- DMR model: $\chi_{\min }^{2}=15.098(\mathrm{AIC}=19.598), \gamma=$ $0.640 \pm 0.066$ and $\log \left(M_{h} / h^{-1} M_{\odot}\right)=12.730 \pm 0.074$.

- For BPR model: $\chi_{\min }^{2}=17.048(\mathrm{AIC}=21.548), \gamma=$ $0.640 \pm 0.075$ and $\log \left(M_{h} / h^{-1} M_{\odot}\right)=13.080 \pm 0.073$.

We observe that the aforementioned bias models provide very similar results (within $1 \sigma$ errors) as far as the growth index is concerned. The corresponding best fit values show a small but non-zero deviation from the theoretically predicted value of GR $\gamma_{\mathrm{GR}} \approx 6 / 11$ (see solid lines of Fig. 1), where the range of the confidence level is $\sim 1.3 \sigma-1.7 \sigma$. Such a small discrepancy between the predicted and observationally fitted value of $\gamma$ has also been discussed by other authors. For example recently, Zhao et al. [70] found $\gamma=0.656_{-0.046}^{+0.042}$, while [69] obtained $\gamma=0.628_{-0.039}^{+0.036}$. Also, similar results can be found in previous papers $[14,29,49,68]$ in which the tension can reach to $\sim 2.5 \sigma$.

Furthermore, we find that the best bias model is the SMT, however the inequality $\triangle \mathrm{AIC} \leq 2$ indicates that the SMT bias model is statistically equivalent with rest of the models. The second result is that the differences of the bias models are absorbed in the fitted value of the DM halo mass in which LRGs live, and which ranges from $\sim 6.2 \times 10^{12} h^{-1} M_{\odot}-1.2 \times 10^{13} h^{-1} M_{\odot}$, for the different bias models and in the case of DESY1COSMO bias. As it can also be seen from Table 2, our derived mass of the host DM halo mass is consistent with that of Papageorgiou et al. [44], while Sawangwit et al. [50] and Pouri et al. [47] found $M_{h} \simeq(1.9-2) \times 10^{13} h^{-1} M_{\odot}$.

In order to complete the present investigation we repeat the likelihood procedure in the case of Planck TT+TE+EE+ low+lensing $\Lambda \mathrm{CDM}$ cosmology, hence $\Omega_{m 0}=1-\Omega_{\Lambda 0}=$ $0.3153, h=0.6736, \Omega_{b 0}=0.0493, n=0.9649$, and $\sigma_{8}=0.811$ [2]. Specifically, for the explored bias models we obtain (see also Table 2):

- For SMT model: $\chi_{\min }^{2}=15.057(\mathrm{AIC}=19.557), \gamma=$ $0.680 \pm 0.076$ and $\log \left(M_{h} / h^{-1} M_{\odot}\right)=13.070 \pm 0.064$.

- For JING model: $\chi_{\min }^{2}=15.952(\mathrm{AIC}=20.452), \gamma=$ $0.690 \pm 0.071$ and $\log \left(M_{h} / h^{-1} M_{\odot}\right)=12.970 \pm 0.058$. 
Table 2 Observational constraints for the joint analysis of bias (see Table 1) and growth rate data: The 1st column shows the expansion model (see Sect. 2; Abbott et al. [1]), the 2nd column indicates the bias models (see Sect. 3), the 3rd column corresponds to $\gamma$ and the $4^{r t h}$ provides the fitted DM halo mass. The remaining columns present the goodness-of-fit statistics $\chi_{\min }^{2}, \mathrm{AIC}$ and $\Delta \mathrm{AIC}=\mathrm{AIC}_{\mathrm{i}}-\mathrm{AIC}_{\min }$. The index $i$ corresponds to the indicated bias model

\begin{tabular}{|c|c|c|c|c|c|c|}
\hline$\Lambda \mathrm{CDM}$ expansion model & Bias model & $\gamma$ & $\log \left(M / h^{-1} M_{\odot}\right)$ & $\chi_{\min }^{2}$ & AIC & $\triangle \mathrm{AIC}$ \\
\hline \multirow{4}{*}{$\begin{array}{l}\text { DES/Planck/JLA/BAO } \\
\text { Abbott et al. [1] }\end{array}$} & SMT & $0.640 \pm 0.071$ & $13.000 \pm 0.072$ & 15.042 & 19.542 & 0 \\
\hline & JING & $0.650 \pm 0.063$ & $12.910 \pm 0.062$ & 15.975 & 20.475 & 0.933 \\
\hline & DMR & $0.640 \pm 0.066$ & $12.730 \pm 0.074$ & 15.096 & 19.598 & 0.056 \\
\hline & BPR & $0.640 \pm 0.075$ & $13.080 \pm 0.073$ & 17.048 & 21.548 & 2.006 \\
\hline \multirow{4}{*}{$\begin{array}{l}\text { Planck TT + TE + EE + low + } \\
\text { lensing Aghanim et al. [2] }\end{array}$} & SMT & $0.680 \pm 0.076$ & $13.07 \pm 0.064$ & 15.057 & 19.557 & 0 \\
\hline & JING & $0.690 \pm 0.071$ & $12.97 \pm 0.058$ & 15.952 & 20.452 & 0.895 \\
\hline & DMR & $0.680 \pm 0.075$ & $12.80 \pm 0.073$ & 15.104 & 19.604 & 0.047 \\
\hline & BPR & $0.680 \pm 0.089$ & $13.06 \pm 0.080$ & 16.947 & 21.447 & 18.49 \\
\hline
\end{tabular}
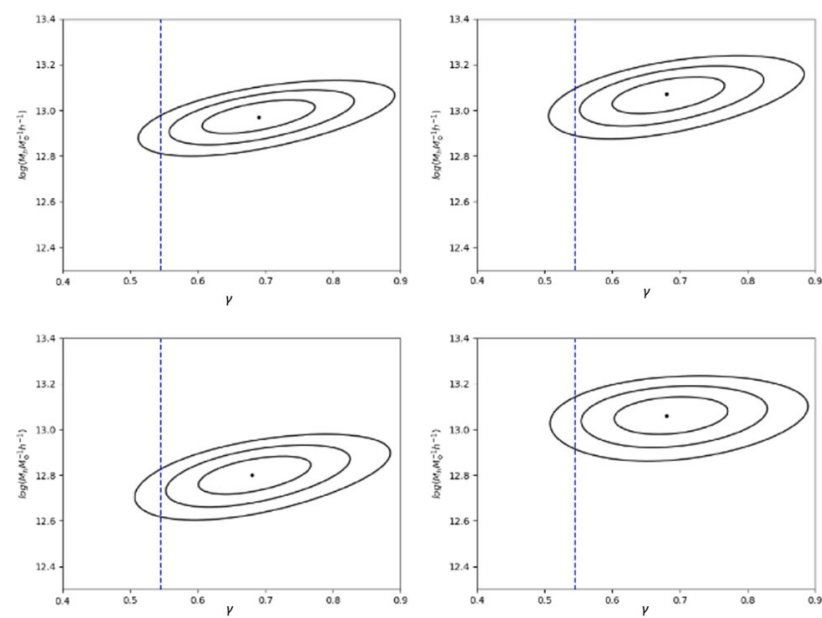

Fig. 1 The iso-likelihood contours for $1 \sigma-2 \sigma-3 \sigma$ levels in the $\left(\gamma, M_{h}\right)$ parameter space for different bias models. Upper row: From left to right, JING and SMT models. Lower row: DMR and BPR models. For further details regarding the models, please see the relevant section. Notice that we use the DES/Planck/JLA/BAO $\Lambda \mathrm{CDM}$ cosmology [1]. The best fit values are given in Table 2. The vertical dashed line corresponds to $\gamma_{\mathrm{GR}} \sim 6 / 11$

- DMR model: $\chi_{\min }^{2}=15.104(\mathrm{AIC}=19.604), \gamma=$ $0.680 \pm 0.075$ and $\log \left(M_{h} / h^{-1} M_{\odot}\right)=12.800 \pm 0.073$.

- For BPR model: $\chi_{\min }^{2}=16.947(\mathrm{AIC}=21.447), \gamma=$ $0.680 \pm 0.089$ and $\log \left(M_{h} / h^{-1} M_{\odot}\right)=13.060 \pm 0.080$.

Obviously, our statistical results remain quite robust (within $1 \sigma$ ) against the choice of the undelying expansion $[1,2]$. Moreover, as it can be seen from Fig. 2 the growth index of the Planck TT $+\mathrm{TE}+\mathrm{EE}+$ low + lensing $\Lambda \mathrm{CDM}$ cosmology deviates with respect to that of GR $\left(\gamma_{\mathrm{GR}} \approx 6 / 11\right)$ at $\sim 1.5-2 \sigma$ levels.
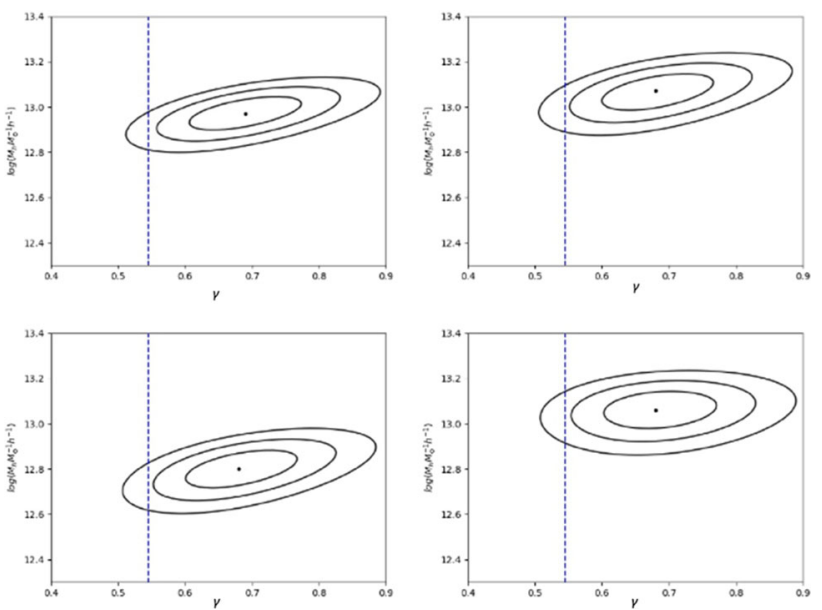

Fig. 2 The iso-likelihood contours for $1 \sigma-2 \sigma-3 \sigma$ levels in the $\left(\gamma, M_{h}\right)$ parameter space for different bias models. Upper row: From left to right, JING and SMT models. Lower row: DMR and BPR models. For further details regarding the models, please see the relevant section. Notice that we use the Planck TT+TE+EE+low+lensing $\Lambda$ CDM cosmology [2]. The best fit values are given in Table 2 . The vertical dashed line corresponds to $\gamma_{\mathrm{GR}} \approx 6 / 11$

\subsubsection{Constraints on $\gamma(z)$}

In this section we implement the overall likelihood procedure in the $\left(\gamma_{0}, \gamma_{1}\right)$ parameter space. Based on the considerations discussed in the previous section the statistical vector takes the form $\mathbf{p}_{1}=\left\{\Omega_{m 0}, h, \sigma_{8}, \gamma_{0}, \gamma_{1}, M_{h}\right\}$.

In Fig. 3 we plot the results of our statistical analysis in the $\left(\gamma_{0}, \gamma_{1}\right)$ plane for the SMT bias model, since we have verified that using the other bias models we get similar contours. The predicted $\left(\gamma_{0}^{(t h)}, \gamma_{1}^{(t h)}\right) \Lambda \mathrm{CDM}$ values are indicated by the solid point, while the star corresponds to our best fit values. In brief for the DES/Planck/JLA/BAO $\Lambda$ cosmology we find $\gamma_{0}=0.630 \pm 0.072, \gamma_{1}=0.040 \pm 0.403$ with 

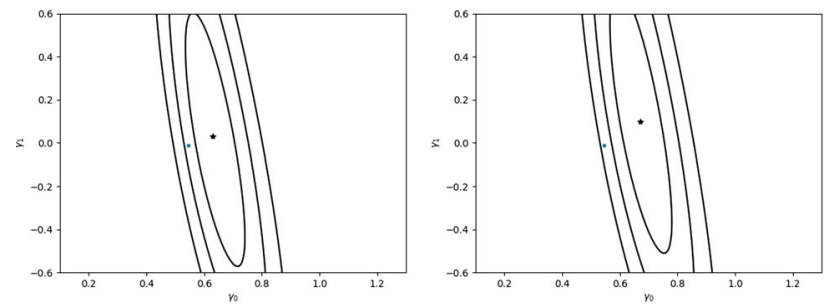

Fig. 3 Iso-likelihood contours for $\Delta \chi^{2}=-2 \ln \mathcal{L} / \mathcal{L}_{\max }$ equal to 2.30, 6.18 and 11.83, corresponding to $1 \sigma, 2 \sigma$ and $3 \sigma$ confidence levels in the $\left(\gamma_{0}, \gamma_{1}\right)$ plane in the case of $\Gamma_{1}$ parametrization. The bias model is that of SMT, while the star corresponds to the best-fit point and the dot to the theoretical $\Lambda \mathrm{CDM}$ point $\left(\gamma_{0}, \gamma_{1}\right)=(0.556,-0.011)$. In the right panel we present the contours that obtained using the Planck TT $+\mathrm{TE}+\mathrm{EE}+$ low + lensing $\Lambda \mathrm{CDM}$ cosmology [2], while in the left the contours obtained using DES/Planck/JLA/BAO $\Lambda$ CDM cosmology

$\chi_{\min }^{2}=15.033(\mathrm{AIC}=19.533)$, while in the case of Planck $\mathrm{TT}+\mathrm{TE}+\mathrm{EE}+$ low + lensing $\Lambda \mathrm{CDM}$ cosmology we get $\gamma_{0}=0.670 \pm 0.073, \gamma_{1}=0.100 \pm 0.422$ with $\chi_{\min }^{2}=$ 14.988 (AIC=19.488). Notice that for the sake of simplicity we have marginalized the likelihood analysis over the LRG dark matter halo, namely $\log \left(M_{h} / h^{-1} M_{\odot}\right)=13.00$ and 13.07 respectively (see SMT model in Table 2).

We conclude that the joint statistical analysis put tight constraints $\gamma_{0}$, however for $\gamma_{1}$ the corresponding error bars remain quite large. Also the range of deviation from GR is $1.7-2.9 \sigma$. We argue that with the next generation of data (mainly from Euclid) we will be able to test whether the growth index of matter fluctuations depends on time.

\section{Conclusions}

In the context of the concordance $\Lambda$ CDM model, testing the validity of general relativity (GR) on extragalactic scales is considered one of the most important tasks in cosmological studies, hence it is crucial to minimize the amount of priors needed to successfully complete such an effort. One such prior is the growth index $(\gamma)$ of matter perturbations. It is well known that a necessary step toward testing GR is to measure $\gamma$ at the $\sim 1 \%$ accuracy level. Obviously, in order to control the systematic effects that possibly affect individual methods and tracers of the growth of matter perturbations we need to have independent estimations of $\gamma$.

Despite the fact that the $\Lambda \mathrm{CDM}+\mathrm{GR}$ model is found to be in a very good agreement with the majority of cosmological data [2], nonetheless the model seems to be currently in tension with some recent measurements, ${ }^{3}$ related with the Hubble constant $H_{0}$ and the present value of the mass variance at $8 h^{-1} \mathrm{Mpc} \sigma_{8}$. Whether these tensions are the result of yet unknown systematic errors or hint some underlying

\footnotetext{
${ }^{3}$ For recent reviews see $[54,64]$.
}

new Physics is still unclear. In the light of the latter results, an intense debate is taking place in the literature and the aim of the present article is to contribute to this debate.

In this work we used the biasing properties of the Luminous Red Galaxies, recently released by the group of Dark Energy Survey (DES), together with growth rate data in order to constrain the growth index of matter perturbations. Specifically, in the framework of concordance $\Lambda+$ GR cosmology, we study the ability of four bias models to fit the DES bias data. Then we combined bias in a joint analysis with the growth rate of matter fluctuations to place constraints on the parameters.

Considering a constant growth index we placed constraints, up to $\sim 10 \%$ accuracy, on the growth index. Specifically, using the priors of the Dark Energy Survey we found that the constraints remain mostly unaffected by using different forms of bias. In particular, we obtained $0.640 \pm 0.071,0.650 \pm 0.063,0.640 \pm 0.066$ and $\gamma=$ $0.640 \pm 0.075$ for SMT [51], JING [34], DMR [21] and BPR [11] bias models. Also utilizing the Planck priors we got $\gamma=0.680 \pm 0.076, \gamma=0.690 \pm 0.071, \gamma=0.680 \pm 0.075$ and $\gamma=0.680 \pm 0.089$ for the aforementioned bias factors. Obviously, we found a small but non-zero deviation from GR $\left(\gamma_{\mathrm{GR}} \approx 6 / 11\right)$, where the confidence level lies in the interval $\sim 1.3 \sigma-2 \sigma$. Such a small discrepancy between the predicted and observationally fitted value of $\gamma$ has also been reported in several studies [14,29,49,68-70]. Moreover, the intrinsic differences of the bias models are absorbed in the fitted value of the dark-matter halo mass in which LRGs survive, and which belongs in the range $\sim 6.2 \times 10^{12} h^{-1} M_{\odot}-1.2 \times 10^{13} h^{-1} M_{\odot}$.

Under the assumption that the growth index varies with time, namely $\gamma(z)=\gamma_{0}+\gamma_{1} z /(1+z)$, we showed that the $\left(\gamma_{0}, \gamma_{1}\right)$ parameter solution space accommodates the GR $\left(\gamma_{0}, \gamma_{1}\right)$ values at $\sim 1.7 \sigma(\sim 2.9 \sigma)$ level utilizing the DES/Planck/JLA/BAO (Planck) priors. Similar to previous studies, we placed tight constraints on $\gamma_{0}$, however the corresponding uncertainties of $\gamma_{1}$ remain large. The next generation (mainly from Euclid) of dynamical data are expected to improve the constraints on $\gamma_{1}$, hence the validity of general relativity on extragalactic scales will be effectively checked.

Acknowledgements Spyros Basilakos acknowledges support from the Cyprus Research Promotion Foundation in the context of the program GRATOS: Graph Theoretical Tools for Sciences (ref. number EXCELLENCE/216/0207) led by European University Cyprus.

Data Availability Statement This manuscript has no associated data or the data will not be deposited. [Authors' comment: We used data from Refs. [23,48].]

Open Access This article is licensed under a Creative Commons Attribution 4.0 International License, which permits use, sharing, adaptation, distribution and reproduction in any medium or format, as long as you give appropriate credit to the original author(s) and the source, pro- 
vide a link to the Creative Commons licence, and indicate if changes were made. The images or other third party material in this article are included in the article's Creative Commons licence, unless indicated otherwise in a credit line to the material. If material is not included in the article's Creative Commons licence and your intended use is not permitted by statutory regulation or exceeds the permitted use, you will need to obtain permission directly from the copyright holder. To view a copy of this licence, visit http://creativecomm ons.org/licenses/by/4.0/.

Funded by SCOAP ${ }^{3}$.

\section{Appendix}

In this appendix we examine the nonlinear power spectrum of [60]. These authors, based on high resolution N-body simulations they improved the original halofit model of [53] (see also $[33,59,63])$. The halo-fit model of [60] is applicable in the wave-number range of $k<30 \mathrm{~h} \mathrm{Mpc}^{-1}$ and the redshift range of $0 \leq z \leq 10$.

In brief, the nonlinear power spectrum consists of two terms:

$\Delta^{2}(k, z)=\Delta_{H}^{2}(k, z)+\Delta_{Q}^{2}(k, z)$

where $\Delta^{2}(k)$ is the dimensionless nonlinear power spectrum $\Delta^{2}(k)=k^{3} P(k) /\left(2 \pi^{2}\right)$. The first term in the above formula is the so called one-halo term which dominates the power spectrum at small scales and it describes the matter correlation within the same dark matter halo. Also, the second is the two-halo term, which dominates the power spectrum at large scales and it describes the matter correlation between two distinct halos.

The one-halo term is given by:

$\Delta_{H}^{2}(k)=\frac{\Delta_{H}^{\prime 2}(k)}{1+\mu_{n} y^{-1}+v_{n} y^{-2}}$

where

$\Delta_{H}^{\prime 2}(k)=\frac{a_{n} y^{3 f_{1}\left(\Omega_{m}\right)}}{1+b_{n} y^{f_{2}\left(\Omega_{m}\right)}+\left[c_{n} f_{3}\left(\Omega_{m}\right) y\right]^{3-\gamma_{n}}}$

with $f_{1}\left(\Omega_{m}\right)=\Omega_{m}^{-0.0307}(z), f_{2}\left(\Omega_{m}\right)=\Omega_{m}^{-0.0585}(z)$, $f_{3}\left(\Omega_{m}\right)=\Omega_{m}^{-0.0743}(z)$. The variable $y$ is the dimensionless wavenumber, $y=k / k_{\sigma}$, where the nonlinear scale, $k_{\sigma}^{-1}=R_{\sigma}$, is defined from the equation $\sigma\left(k_{\sigma}^{-1}, z\right) \equiv 1$ [53], where the Gaussian filter is written as

$\sigma^{2}(R, z)=\int d \ln k \Delta_{L}^{2}(k, z) \exp \left(-k^{2} R^{2}\right)$.

Notice that $\Delta_{L}^{2}(k, z)$ is the linear dimensionless power spectrum $\Delta_{L}^{2}(k, z)=k^{3} P_{L}(k, z) /\left(2 \pi^{2}\right)$ and $P_{L}(k, z)$ is the CDM linear power spectrum given by $P_{L}(k, z)=$ $P_{0} k^{n} T^{2}(k) D^{2}(z)$ where $n$ is the spectral index of the primordial power spectrum and $T(k)$ is the CDM transfer function [22].
Now, following the notations of [60], the two-halo term is given by

$\Delta_{Q}^{2}(k)=\Delta_{L}^{2}(k)\left[\frac{1+\Delta_{L}^{2}(k)^{\beta_{n}}}{1+\alpha_{n} \Delta_{L}^{2}(k)}\right] \exp ^{-f(y)}$

with $f(y)=y / 4+y^{2} / 8$. Notice, that the free parameters of the model, namely $a_{n}, b_{n}, c_{n}, \gamma_{n}, \alpha_{n}, \beta_{n}, \mu_{n}$ and $v_{n}$ can be found in [60]. These parameters are given in terms of the effective spectral index $n_{\text {eff }}$ and the curvature $C$ defined as:

$n_{\mathrm{eff}}+3=-\left.\frac{d \ln \sigma^{2}(R)}{d \ln R}\right|_{\sigma=1}$

and

$C=-\left.\frac{d^{2} \ln \sigma^{2}(R)}{d \ln R^{2}}\right|_{\sigma=1}$.

\section{References}

1. B.P. Abbott et al., Phys. Rev. D 97, 102002 (2018). https://doi.org/ 10.1103/PhysRevD.97.102002

2. N. Aghanim et al. [Planck Collaboration]. arXiv:1807.06209 [astro-ph.CO]

3. H. Akaike, IEEE Trans. Autom. Control 19, 716 (1974)

4. L. Amendola, R. Catena, I. Masina, A. Notari, M. Quartin, C. Quercellini, JCAP 1107, 027 (2011). https://doi.org/10.1088/ 1475-7516/2011/07/027

5. G. Ballesteros, A. Riotto, Phys. Lett. B 668, 171 (2008). https:// doi.org/10.1016/j.physletb.2008.08.035

6. S. Basilakos, Phys. Rev. D 93, 083007 (2016). https://doi.org/10. 1103/PhysRevD.93.083007

7. S. Basilakos, S. Nesseris, Phys. Rev. D 94, 123525 (2016). https:// doi.org/10.1103/PhysRevD.94.123525

8. S. Basilakos, M. Plionis, Mon. Not. R. Astron. Soc 360, 35 (2005). https://doi.org/10.1111/j.1745-3933.2005.00041.x

9. S. Basilakos, A. Pouri, Mon. Not. R. Astron. Soc 423, 3761 (2012). https://doi.org/10.1111/j.1365-2966.2012.21168.x

10. S. Basilakos, J. Solà, Phys. Rev. D 92, 123501 (2015). https://doi. org/10.1103/PhysRevD.92.123501

11. S. Basilakos, J.B. Dent, S. Dutta, L. Perivolaropoulos, M. Plionis, Phys. Rev. D 85, 123501 (2012). https://doi.org/10.1103/ PhysRevD.85.123501

12. S. Basilakos, A.P. Kouretsis, E.N. Saridakis, P. Stavrinos, Phys. Rev. D 88, 123510 (2013). https://doi.org/10.1103/PhysRevD.88. 123510

13. M. Betoule et al., Astron. Astrophys. 568, A22 (2014). https://doi. org/10.1051/0004-6361/201423413

14. F. Beutler et al., Mon. Not. R. Astron. Soc. 444, 3501 (2014). https://doi.org/10.1093/mnras/stu 1702

15. C. Blake et al., Mon. Not. R. Astron. Soc. 418, 1707 (2011). https:// doi.org/10.1111/j.1365-2966.2011.19592.x

16. B.A. Bueno, J. Garcia-Bellido, D. Sapone, JCAP 1110, 010 (2011) https://doi.org/10.1088/1475-7516/2011/10/010

17. K.P. Burnham, D.R. Anderson, Sociol. Methods Res. 33, 261 (2004)

18. R. Caldwell, M. Kamionkowski, Nature 458, 587 (2009). https:// doi.org/10.1038/458587a

19. P. Catelan, F. Lucchin, S. Matarrese, C. Porciani, Mon. Not. R. Astron. Soc. 297, 692 (1998). https://doi.org/10.1046/j. 1365-8711.1998.01455.x 
20. E.J. Copeland, M. Sami, S. Tsujikawa, Int. J. Mod. Phys. D 15, 1753 (2006). https://doi.org/10.1142/S021827180600942X

21. A. De Simone, M. Maggiore, A. Riotto, Mon. Not. R. Astron. Soc. 418, 2403 (2011). https://doi.org/10.1111/j.1365-2966.2011. 19638.x

22. D.J. Eisenstein, W. Hu, Astrophys. J. 511, 5 (1997). https://doi.org/ $10.1086 / 306640$

23. J. Elvin-Poole et al., Phys. Rev. D 98, 042006 (2018). https://doi. org/10.1103/PhysRevD.98.042006

24. O. Farooq, S. Crandall, B. Ratra, Phys. Lett. B 726, 72 (2013). https://doi.org/10.1016/j.physletb.2013.08.078

25. J.N. Fry, Astrophys. J. 461, L65 (1996). https://doi.org/10.1086/ 310006

26. X.-Y. Fu, P.-X. Wu, H.-W. Yu, Phys. Lett. B 677, 12 (2009). https:// doi.org/10.1016/j.physletb.2009.05.007

27. R. Gannouji, B. Moraes, D. Polarski, JCAP 02, 034 (2009). https:// doi.org/10.1088/1475-7516/2009/02/034

28. R. Gannouji, L. Kazantzidis, L. Perivolaropoulos, D. Polarski, Phys. Rev. D 98, 104044 (2018). https://doi.org/10.1103/ PhysRevD.98.104044

29. H. Gil-Marín, W.J. Percival, L. Verde, J.R. Brownstein, C.-H. Chuang, F.-S. Kitaura, S.A. Rodríguez-Torres, M.D. Olmstead, Mon. Not. R. Astron. Soc. 465, 1757 (2017). https://doi.org/10. 1093/mnras/stw2679

30. Y. Gong, Phys. Rev. D 78, 123010 (2008). https://doi.org/10.1103/ PhysRevD.78.123010

31. M. Hicken, W.M. Wood-Vasey, S. Blondin, P. Challis, S. Jha, P.L. Kelly, A. Rest, R.P. Kirshner, Astrophys. J. 700, 1097 (2009). https://doi.org/10.1088/0004-637X/700/2/1097

32. D. Huterer et al., Astropart. Phys. 63, 23 (2015). https://doi.org/ 10.1016/j.astropartphys.2014.07.004

33. K.T. Inoue, R. Takahashi, Mon. Not. R. Astron. Soc. 426, 2978 (2012). https://doi.org/10.1111/j.1365-2966.2012.21915.x

34. Y.P. Jing, Astrophys. J. 503, L9 (1998). https://doi.org/10.1086/ 311530

35. E. Krause, et al., (DES Collaboration). submitted to Phys. Rev. D) (2017). arXiv:1706.09359 [astro-ph.CO]

36. M. Krumpe, T. Miyaji, A.L. Coil, Acta Polytech. Proc. 1, 71 (2014). https://doi.org/10.14311/APP.2014.01.0071

37. A.R. Liddle, Mon. Not. R. Astron. Soc. 377, L74 (2007). https:// doi.org/10.1111/j.1745-3933.2007.00306.x

38. E.V. Linder, Astropart. Phys. 29, 336 (2008). https://doi.org/10. 1016/j.astropartphys.2008.03.002

39. E.V. Linder, R.N. Cahn, Astropart. Phys. 28, 481 (2007). https:// doi.org/10.1016/j.astropartphys.2007.09.003

40. T. Matsubara, A.S. Szalay, A.C. Pope, Astrophys. J. 606, 1 (2004). https://doi.org/10.1086/382783

41. A. Mehrabi, S. Basilakos, M. Malekjani, Z. Davari, Phys. Rev. D 92, 123513 (2015). https://doi.org/10.1103/PhysRevD.92.123513

42. S. Nesseris, Phys. Rev. D 79, 044015 (2009). https://doi.org/10. 1103/PhysRevD.79.044015

43. S. Nesseris, D. Sapone, Phys. Rev. D 92, 023013 (2015). https:// doi.org/10.1103/PhysRevD.92.023013

44. A. Papageorgiou, S. Basilakos, M. Plionis, Mon. Not. R. Astron. Soc. 476, 2621 (2018). https://doi.org/10.1093/mnras/sty396
45. P.J.E. Peebles, Principles of Physical Cosmology (Princeton University Press, Princeton, New Jersey, 1993)

46. D. Polarski, R. Gannouji, Phys. Lett. B 660, 439 (2008). https:// doi.org/10.1016/j.physletb.2008.01.032

47. A. Pouri, S. Basilakos, M. Plionis, JCAP 1408, 042 (2014). https:// doi.org/10.1088/1475-7516/2014/08/042

48. B. Sagredo, S. Nesseris, D. Sapone, Phys. Rev. D 98, 083543 (2018). https://doi.org/10.1103/PhysRevD.98.083543

49. L. Samushia et al., Mon. Not. R. Astron. Soc. 439, 3504 (2014). https://doi.org/10.1093/mnras/stu197

50. U. Sawangwit, T. Shanks, S.M. Croom, M.J. Drinkwater, S. Fine, D. Parkinson, N.P. Ross, Mon. Not. R. Astron. Soc. 2012, 420 (1916). https://doi.org/10.1111/j.1365-2966.2011.19848.x

51. R.K. Sheth, G. Tormen, Mon. Not. R. Astron. Soc. 308, 119 (1999). https://doi.org/10.1046/j.1365-8711.1999.02692.x

52. V. Silveira, I. Waga, Phys. Rev. D 50, 4890 (1994). https://doi.org/ 10.1103/PhysRevD.50.4890

53. R.E. Smith et al., Mon. Not. R. Astron. Soc. 341, 1311 (2003). https://doi.org/10.1046/j.1365-8711.2003.06503.x

54. P.J. Solà, Int. J. Mod. Phys. A 33, 1844009 (2018)

55. Y.-S. Song, W.J. Percival, JCAP 0910, 004 (2009). https://doi.org/ 10.1088/1475-7516/2009/10/004

56. H.F. Stabenau, B. Jain, Phys. Rev. D 74, 084007 (2006). https:// doi.org/10.1103/PhysRevD.74.084007

57. H. Steigerwald, J. Bel, C. Marinoni, JCAP 1405, 042 (2014). https://doi.org/10.1088/1475-7516/2014/05/042

58. N. Sugiyama, J. Suppl. Astrophys 100, 281 (1995). https://doi.org/ $10.1086 / 192220$

59. R. Takahashi, M. Oguri, M. Sato, T. Hamana, Astrophys. J. 742, 15 (2011). https://doi.org/10.1088/0004-637X/742/1/15

60. R. Takahashi, M. Sato, T. Nishimichi, A. Taruya, M. Oguri, Astrophys. J. 761, 152 (2012). https://doi.org/10.1088/0004-637X/761/ $2 / 152$

61. S. Tsujikawa, R. Gannouji, B. Moraes, D. Polarski, Phys. Rev. D 80, 084044 (2009). https://doi.org/10.1103/PhysRevD.80.084044

62. J.-P. Uzan, F. Bernardeau, Y. Mellier, Phys. Rev. D 77, 021301 (2008). https://doi.org/10.1103/PhysRevD.77.021301

63. P. Valageas, T. Nishimichi, Astron. Astrophys. 527, A87 (2011). https://doi.org/10.1051/0004-6361/201015685

64. L. Verde, T. Treu, A. G. Riess, Nat. Astron. (2019) [arXiv:1907.10625]. https://doi.org/10.1038/s41550-019-0902-0

65. L.-M. Wang, P.J. Steinhardt, Astrophys. J. 508, 483 (1998). https:// doi.org/10.1086/306436

66. H. Wei, Phys. Lett. B 664, 1 (2008). https://doi.org/10.1016/j. physletb.2008.04.060

67. N.N. Weinberg, M. Kamionkowski, Mon. Not. R. Astron. Soc. 341, 251 (2003). https://doi.org/10.1046/j.1365-8711.2003.06421.x

68. L. Xu, Phys. Rev. D 88, 084032 (2013). https://doi.org/10.1103/ PhysRevD.88.084032

69. Z.-Y. Yin, H. Wei, Eur. Phys. J. C 79, 698 (2019). https://doi.org/ 10.1140/epjc/s10052-019-7191-8

70. M.-M. Zhao, J.-F. Zhang, X. Zhang, Phys. Lett. B 779, 473 (2018). https://doi.org/10.1016/j.physletb.2018.02.042 